\title{
Utilizing Internal Standard Responses to Assess Risk on Reporting Bioanalytical Results from Hemolyzed Samples
}

\author{
Eliza N. Fung, ${ }^{1,2}$ Anne-Françoise Aubry, ${ }^{1}$ Alban Allentoff, ${ }^{1}$ and Qin C. Ji ${ }^{1,2}$
}

Received 20 March 2015; accepted 4 May 2015; published online 15 May 2015

\begin{abstract}
Bioanalytical analysis of toxicokinetic and pharmacokinetic samples is an integral part of small molecule drugs development and liquid chromatography-tandem mass spectrometry (LC-MS/MS) has been the technique of choice. One important consideration is the matrix effect, in which ionization of the analytes of interest is affected by the presence of co-eluting interfering components present in the sample matrix. Hemolysis, which results in additional endogenous components being released from the lysed red blood cells, may cause additional matrix interferences. The effects of the degree of hemolysis on the accuracy and precision of the method and the reported sample concentrations from hemolyzed study samples have drawn increasing attention in recent years, especially in cases where the sample concentrations are critical for pharmacokinetic calculation. Currently, there is no established procedure to objectively assess the risk of reporting potentially inaccurate bioanalytical results from hemolyzed study samples. In this work, we evaluated the effect of different degrees of hemolysis on the internal standard peak area, accuracy, and precision of the analyses of BMS-906024 and its metabolite, BMS911557, in human plasma by LC-MS/MS. In addition, we proposed the strategy of using the peak area of the stable isotope-labeled internal standard (SIL-IS) from the LC-MS/MS measurement as the surrogate marker for risk assessment. Samples with peak areas outside of the pre-defined acceptance criteria, e.g., less than $50 \%$ or more than $150 \%$ of the average IS response in study samples, plasma standards, and QC samples when SIL-IS is used, are flagged out for further investigation.
\end{abstract}

KEY WORDS: hemolyzed sample/haemolyzed sample; hyperlipemic sample; internal standard peak area/internal standard responses; LC-MS/MS; regulated bioanalysis; risk assessment.

\section{INTRODUCTION}

Bioanalytical analysis of toxicokinetic and pharmacokinetic samples is an integral part of drug development. Liquid chromatography-tandem mass spectrometry (LC-MS/MS) in which low molecular weight analytes $(<2 \mathrm{kD})$ are separated from each other and from the endogenous components by liquid chromatography and then detected by tandem mass spectrometry has been the technique of choice for the past two decades (1-6). One of the important considerations during mass spectrometric detection is the presence of matrix effect, in which ionization of the analytes of interest can be affected by the presence of co-eluting interfering components present in the sample matrix (7-12). Matrix effect, in the form of ion suppression, or in some cases, ion enhancement, is a well-recognized phenomenon that affects bioanalysis by LC-MS/MS. The matrix factor, on the other hand, is the quantitative measurement of the matrix effect, and different methodologies have been proposed to evaluate this parameter $(8,13-16)$. The determination of

\footnotetext{
${ }^{1}$ Research \& Development, Bristol-Myers Squibb Co., Route 206/ Province Line Road, Lawrenceville, New Jersey 08543, USA.

${ }^{2}$ To whom correspondence should be addressed. (e-mail: ngakiteliza.fung@bms.com; qin.ji@bms.com)
}

matrix factor has become a routine part of bioanalytical method validation.

Hemolysis, which results in additional endogenous components being released from the lysed red blood cells, may cause additional matrix effects relevant to the analytes of interest. Some regulators, for example, in Europe and Brazil, are recommending the evaluation of matrix factor in hemolyzed plasma in their bioanalytical assay validation guidelines (17,18). Analyses of hemolyzed study samples have been much discussed in the bioanalytical field, with a focus on matrix effect, analyte stability in hemolyzed plasma, and "reportability" of the bioanalytical results (19-22). Bioanalytical laboratories in general conduct formal evaluation of matrix factor, specificity/selectivity in hemolyzed plasma during method validation, usually at a pre-defined level of hemolysis, for example, plasma spiked with hemolyzed blood to a final percentage of $2 \%$ (19). In reality, the study samples can be severely hemolyzed with a final percentage well above $2 \%$. It is therefore important to have an in-process evaluation of the impact of extent of hemolysis on the matrix factor, the accuracy and precision of LC-MS/MS-based bioanalytical methods, and most importantly, the reported study sample concentrations. Currently, there is no established procedure to objectively assess the risk of reporting bioanalytical results from hemolyzed study samples. Hence, there is a need to identify an analytical 
parameter to serve as a surrogate marker. Ideally, the analytical parameter should be quantitative, universal, and easy to implement in routine sample analysis.

In this work, we proposed to use the peak area of stable isotope-labeled internal standards (SIL-IS), such as deuterated, ${ }^{13} \mathrm{C}-,{ }^{15} \mathrm{~N}$-labeled analogues from the LC-MS/MS measurement (internal standard responses or IS responses), as the surrogate marker. SIL-IS are generally used to compensate for the impact of matrix effect on the analyte instrument response in each individual sample (23-25). They are chosen for a number of reasons. First, they mimic the mass spectrometric behavior and extraction recovery of the analytes very well. Second, the same amount of internal standard is added to each study sample, plasma standard, and QC sample, and any significant deviation from the average peak area can be calculated with good accuracy. Last, internal standard response is a parameter that is monitored routinely in regulated bioanalysis.

Another challenge lies in defining the extent of hemolysis. In order to investigate the relationship between the extent of hemolysis and matrix effect, accuracy, and precision, it is important to quantitatively express the extent of hemolysis. Hemolysis can be expressed in terms of the concentration of free hemoglobin in a given plasma sample, in $\mathrm{mg} / \mathrm{dL}$, with $\sim 550 \mathrm{mg} / \mathrm{dL}$ corresponding to roughly $2 \%$ of hemolyzed blood in the plasma sample (19) and can be determined spectrophotometrically. Another approach is to estimate the extent of hemolysis in terms of the percentage of hemolyzed blood added to the control plasma (20). Since there are other components besides hemoglobin being released during cell lysis and these components can interfere with the mass spectrometric measurement as well, we decided to express the extent of hemolysis in terms of the percentage of hemolyzed blood added to the control plasma in this work.

We evaluated the effect of different amounts of hemolysis on the matrix factor, accuracy, and precision of the analysis of BMS-906024 and BMS-911557 in human plasma by LCMS/MS. BMS-906024 (Fig. 1a) is currently being developed for treatment of cancer (26), and BMS-911557 is its metabolite (Fig. 1b). The peak areas of their respective SIL-IS, BMS-906024-03 (Fig. 1c), and BMS-911557-02 (Fig. 1d) served as the surrogate markers for evaluation of matrix factor. QC samples of BMS-906024 and BMS-911557 were prepared in plasma with different percentages of hemolyzed blood and analyzed for accuracy and precision evaluation. Herein, we presented the results of the evaluation, the proposed strategy to assess the risk of reporting bioanalytical results from hemolyzed samples, and the proposed resolution to significantly reduce the undesirable matrix effect.

\section{MATERIALS AND METHODS}

\section{Chemicals}

Human plasma ( $\mathrm{K}_{2}$ EDTA) and human whole blood ( $\mathrm{K}_{2}$ EDTA) were obtained from Bioreclamation (Hicksville, NY, USA). Reference standards of BMS-906024 and BMS911557 and their internal standards (BMS-906024-03 and BMS911557-02, respectively) were synthesized in-house at BristolMyers Squibb (Princeton, NJ, USA). Acetonitrile, methanol (HPLC grade), tripotassium ethylenediaminetetraacetate
( $\mathrm{K}_{3}$ EDTA), ammonium formate, and methyl tert-butyl ether (MTBE) were obtained from Sigma-Aldrich (St. Louis, MO, USA). Formic acid was obtained from EMD Chemicals (San Diego, CA, USA). All reagents were analytical grade or better. High-purity water was obtained with a Barnstead Nanopure Diamond water purification system (Dubuque, IA, USA).

\section{Preparation of Stock Solutions of BMS-906024 and BMS-911557 and Their Internal Standards}

Stock solutions of BMS-906024 and BMS-911557 were prepared in acetonitrile at $1.00 \mathrm{mg} / \mathrm{mL}$ with two separate weighings. Stock solutions of BMS-906024-03 and BMS911557-02 were prepared in acetonitrile at $1.00 \mathrm{mg} / \mathrm{mL}$. A combined working solution of BMS-906024 and BMS-911557 at $12.5 \mu \mathrm{g} / \mathrm{mL}$ was prepared by diluting the appropriate volumes of the stock solutions with acetonitrile. A combined internal standard working solution (ISWS) of BMS-90602403 and BMS-911557-02 was prepared by diluting the internal standard stock solutions to $12.5 \mathrm{ng} / \mathrm{mL}$ with $20 \%$ acetonitrile $/ 80 \%$ water. All solutions were stored at $4{ }^{\circ} \mathrm{C}$.

\section{LC-MS/MS Analysis by Triple Quadrupole Mass Spectrometer}

A Rheos Allegro Ultra-4x VHPLC pump (Flux Instruments AG, Switzerland) was used for the LC-MS/MS analysis. The column was a Waters Acquity HSS T3, $2.1 \times 50 \mathrm{~mm}, 1.8 \mu \mathrm{m}$ (Waters, Milford, MA, USA). A CTC HTC-PAL autosampler (Leap Technologies, Carboro, NC, USA) equipped with the dynamic-load wash (DLW) was used. Both the VHPLC pump and autosampler were controlled by Analyst ${ }^{\mathrm{TM}}$ 1.5.1 (AB Sciex, Foster City, CA, USA). The mobile phases were $0.1 \%$ formic acid in water (A) and $0.1 \%$ formic acid in acetonitrile (B). The flow rate was $0.6 \mathrm{~mL} / \mathrm{min}$ and column temperature was set at $40^{\circ} \mathrm{C}$. The following gradient elution was used, starting at $40 \% \mathrm{~B}$, then increasing to $60 \% \mathrm{~B}$ in $2.5 \mathrm{~min}$, increasing again to $100 \% \mathrm{~B}$ in $0.1 \mathrm{~min}$, holding at $100 \% \mathrm{~B}$ for $1.3 \mathrm{~min}$, and then lowering back to $40 \% \mathrm{~B}$ in $0.1 \mathrm{~min}$. The total run time was $4.5 \mathrm{~min}$. The autosampler temperature was set at $10^{\circ} \mathrm{C}$. The autosampler wash solutions were $20 \mathrm{mM} \mathrm{K} \mathrm{K}_{3}$ EDTA in $50 \% \mathrm{MeOH} / 50 \%$ $\mathrm{H}_{2} \mathrm{O}$ and $0.5 \%$ formic acid $/ 50 \% \mathrm{MeOH} / 20 \%$ isopropanol/ $29.5 \% \mathrm{H}_{2} \mathrm{O}$.

The samples were analyzed in positive ion mode using the TurboIonSpray ${ }^{\circledR}$ interface (TIS) of TripleQuad 5500 triple quadrupole mass spectrometer. The following MS/MS conditions were used: ionspray voltage (IS), $4500 \mathrm{~V}$; declustering potential (DP), $90 \mathrm{~V}$; collision energy (CE), $45 \mathrm{eV}$; collision cell exit potential (CXP), $10 \mathrm{~V}$; entrance potential (EP), $10 \mathrm{~V}$; source temperature, $500^{\circ} \mathrm{C}$. Nebulizer gas (GS1 and GS2), curtain gas (CUR), and collision gas (CAD) were set to $40,40,35$, and 8 , respectively. The transitions monitored were $m / z, 557.2 \rightarrow m / z, 221.2$ for BMS$906024, m / z \quad 543.2 \rightarrow m / z \quad 207.2$ for BMS-911557, $m / z$ $563.2 \rightarrow m / z, 227.2$ for BMS-906024-03, and $\mathrm{m} / z \quad 549.2 \rightarrow m / z$ 213.2 for BMS-911557-02. The data acquisition was performed using Analyst ${ }^{\mathrm{TM}}$ 1.5.1 (AB Sciex). 

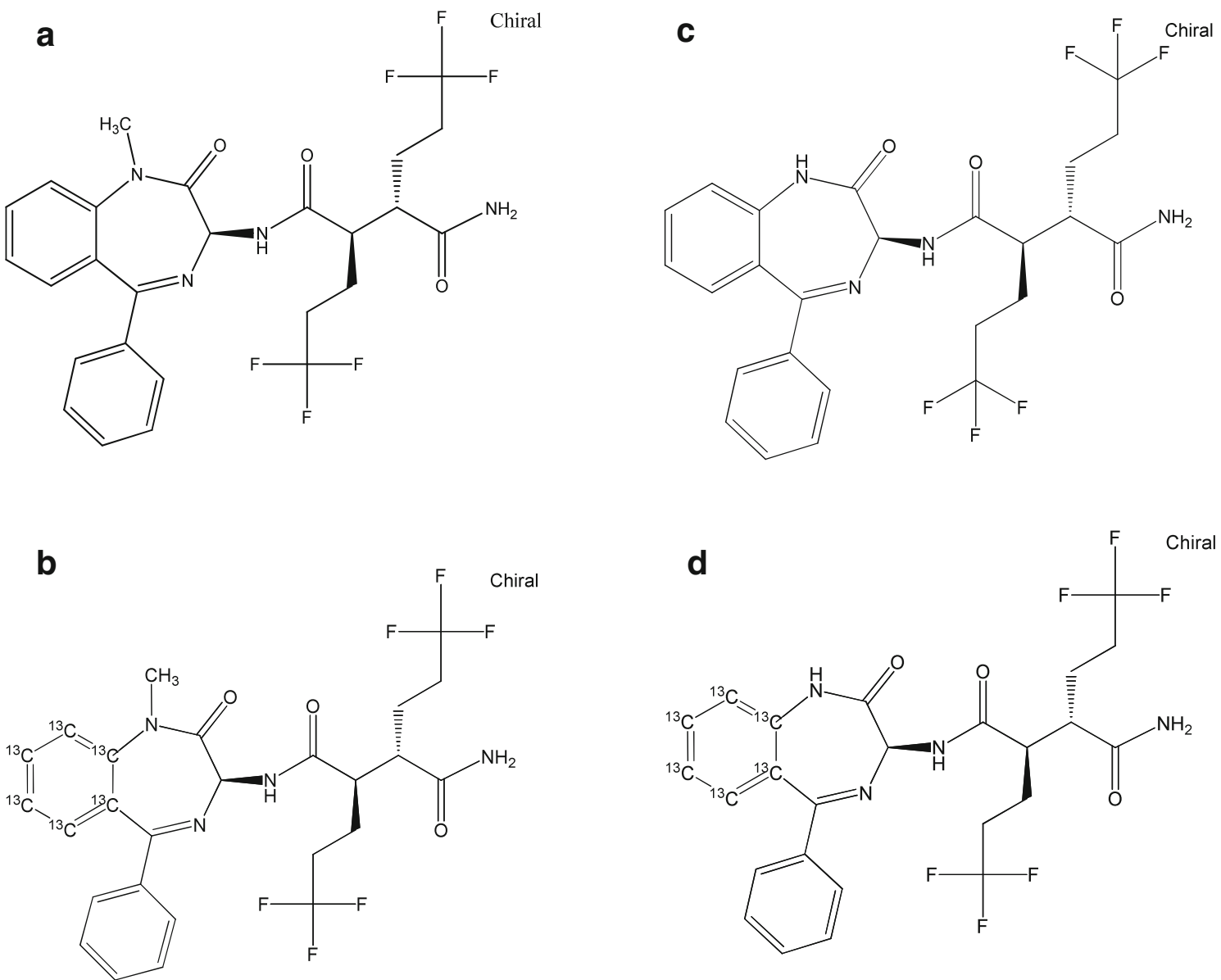

Fig. 1. Structures of a BMS-906024, b BMS-906024-03, c BMS-911557, and d BMS-911557-02

\section{Preparation of Hemolyzed Plasma}

A series of hemolyzed plasma samples $(0.5,1.0,2.0,5.0$, $10.0,20.0,30.0,40.0,50.0 \%)$ was prepared by mixing human plasma with appropriate amount of lysed human whole blood. For example, plasma with $50 \%$ of hemolyzed blood was prepared by mixing $7.5 \mathrm{~mL}$ of human control plasma with $7.5 \mathrm{~mL}$ of lysed human whole blood. Lysed human whole blood was prepared by twice freezing and thawing human whole blood (20). The color of the resulting mixture deepened with increasing percentage of hemolyzed blood, and it is difficult to distinguish beyond $20 \%$ of hemolyzed blood based on color, as depicted in Fig. 2.

\section{Human Plasma Standard and QC Samples of BMS-906024 and BMS-911557}

The calibration curves, consisting of eight concentrations in the range of $0.500-250 \mathrm{ng} / \mathrm{mL}$, were prepared by serial dilution from the $12.5 \mu \mathrm{g} / \mathrm{mL}$ combined working solution of BMS-906024 and BMS-911557 (Section 2.2) with human control plasma. The concentrations of the plasma standards were $0.500,1.00,2.50$, $10.0,40.0,125,200$, and $250 \mathrm{ng} / \mathrm{mL}$. The plasma standards were freshly prepared on the day of use.

For QC samples, the stock solutions of BMS-906024 and BMS-911557 were prepared from separate weighings from those used for the standards. QC samples at 187.5 (high concentration) and $1.50 \mathrm{ng} / \mathrm{mL}$ (low concentration) were prepared by diluting these stock solutions with human control plasma, and the series of hemolyzed plasma prepared in Section 2.4 (1.0, 2.0, 5.0, 10.0, 20.0, $30.0,40.0,50.0 \%)$. The QC samples were stored at $-20^{\circ} \mathrm{C}$.

\section{Liquid-liquid Extraction Procedure for Human Plasma Samples}

The plasma standards and QC samples were extracted by liquid-liquid extraction (LLE) as detailed here. A $100 \mu \mathrm{L}$ volume of sample was mixed with $50 \mu \mathrm{L}$ of ISWS, $50 \mu \mathrm{L}$ of

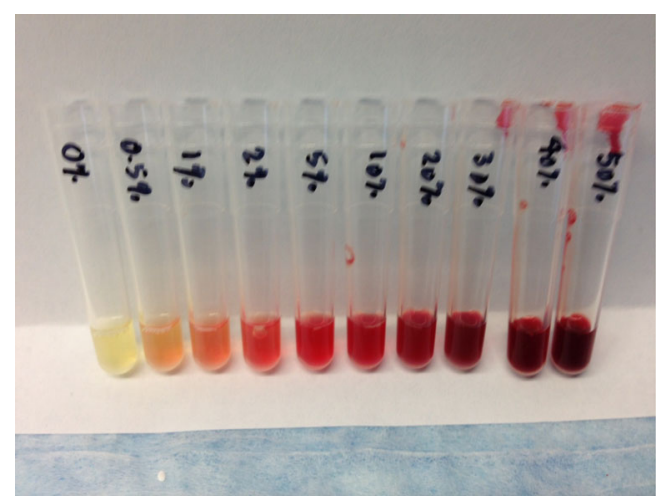

Fig. 2. "Hemolysis chart"-a chart representing plasma samples with different percentages of hemolyzed blood 
$1 \mathrm{M}$ ammonium formate at $\mathrm{pH} 3,650 \mu \mathrm{L}$ of MTBE, followed by shaking for $20 \mathrm{~min}$. The organic layer was separated by centrifugation. Approximately $420 \mu \mathrm{L}$ of the organic layer was transferred to a clean microtube and evaporated to dryness. The extraction recovery was around $70 \%$ for both analytes and their internal standards. The dried extract was reconstituted in $200 \mu \mathrm{L}$ of $40 \%$ acetonitrile/60\% water. A $5-\mu \mathrm{L}$ portion of the reconstituted extract was injected into the LC-MS/MS system.

\section{Evaluation of Internal Standard Responses, Accuracy, and Precision of QC Samples}

Two sets of calibration curves were used to bracket each run. Accuracy and precision were evaluated with triplicate analysis of analytical QC's. In the runs that the high concentration QC's needed to be diluted before analysis, the high concentration QC samples were diluted with appropriate amount of human control plasma.

The peak integration and data regression were performed by Analyst ${ }^{\mathrm{TM}}$ 1.5.1 (AB Sciex). A linear regression with $1 / x^{2}$ weighting was chosen as the regression model. The accuracy was defined as the percentage from nominal concentration. The precision was defined as the \%CV of triplicate analyses of a QC sample.

\section{RESULTS}

Effect of Hemolysis on the Internal Standard Responses, Accuracy, and Precision of the Bioanalytical Method

The low $(1.50 \mathrm{ng} / \mathrm{mL})$ and high $(187.5 \mathrm{ng} / \mathrm{mL})$ concentrations QC samples of BMS-906024 and BMS-911557 prepared in plasma spiked with different percentages of hemolyzed blood (from 1 to $50 \%$ ) were analyzed in triplicate. The QC samples without any hemolyzed blood $(0 \%)$ were similarly analyzed to serve as a reference. The effect of different degrees of hemolysis on the peak areas of the internal standards of BMS-906024 and BMS-911557 (internal standard responses) at low and high QC concentrations is depicted in Fig. 3. With increasing percentages of hemolyzed blood, the average IS responses from triplicate analysis of the QC samples decreased from $1 \%$ of hemolyzed blood to $20 \%$ of hemolyzed blood, with the sharpest drop occurring between $5 \%$ of hemolyzed blood and $20 \%$ of hemolyzed blood, with a relatively constant and low response observed at higher levels of hemolysis $(<1000 \mathrm{cps})$.

The effect of different percentages of hemolyzed blood on the accuracy and precision of analyses of BMS-906024 and BMS-911557 at the low and high QC concentrations is depicted in Figs. 4 and 5, respectively. For BMS-906024, the accuracy was mostly within 85 to $115 \%$ in QC samples containing up to $\sim 30 \%$ of hemolyzed blood, with a slight trend toward decreasing accuracy starting from $20 \%$ of hemolyzed blood. Nevertheless, it met the acceptance criteria based on the Bioanalytical Guidance from US FDA and EU EMA guideline $(18,26)$. The same trends were observed for BMS-911557. The measurement of BMS-906024 and BMS911557 was found to be precise across the range of the

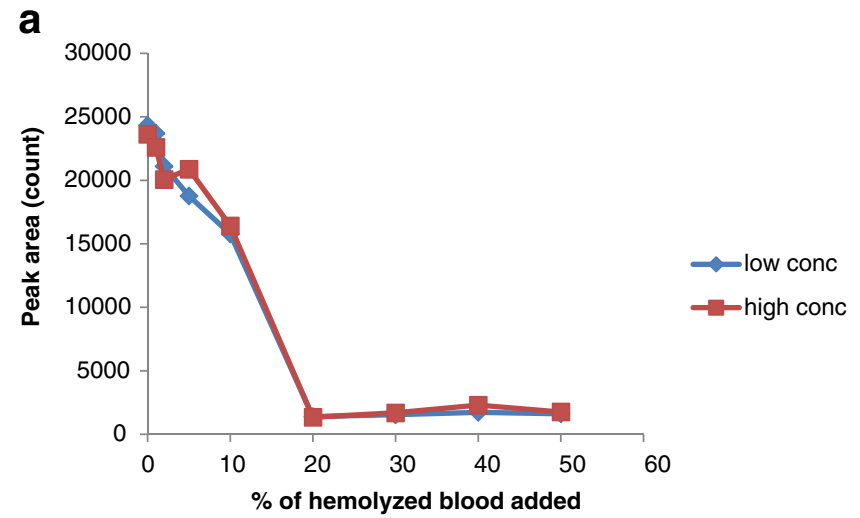

b

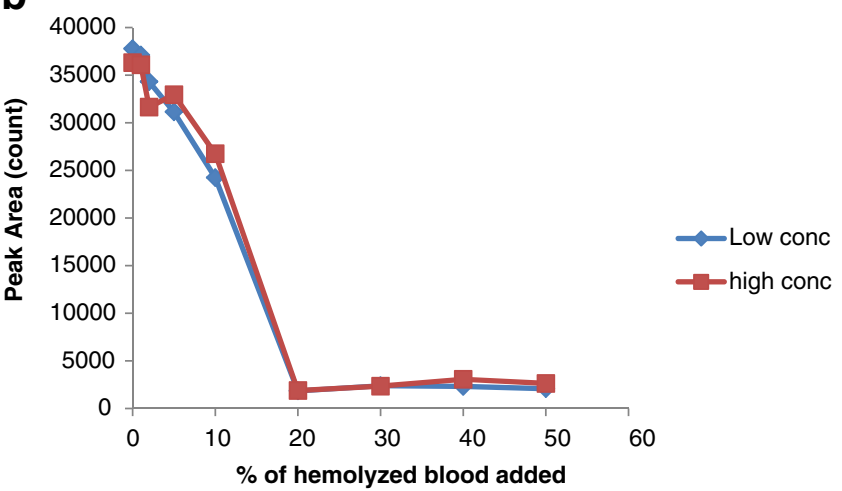

Fig. 3. Average peak area of the internal standards (internal standard responses) of a BMS-906024, b BMS-911557 with different percentages of hemolyzed blood spiked to the plasma. Low conc. is $1.5 \mathrm{ng} / \mathrm{mL}$ and high conc. is $187.5 \mathrm{ng} / \mathrm{mL}$

hemolyzed plasma, with a precision ( $\% \mathrm{CV}$ ) of $<15 \%$ for most concentrations, which also met the regulatory acceptance criteria $(18,27)$. However, on closer inspection, for both BMS-906024 and BMS-911557, the method showed a trend toward lower accuracy and precision starting at a percentage of $\sim 20 \%$ of hemolyzed blood, especially when compared with measurements made at $<10 \%$ or lower of hemolyzed blood, even at high concentration of analytes.

\section{Effect of Dilution on the Internal Standard Responses}

The high concentration QC sample (187.5 ng/mL) prepared in plasma spiked with $40 \%$ of hemolyzed blood was analyzed in triplicate following dilution with human control plasma. Different dilution factors ranging from 1.5 times to 40 times were investigated. The undiluted QC sample was similarly analyzed in triplicate to serve as a reference. The effect of different dilution factors on the average IS responses at low and high QC concentrations is depicted in Fig. 6. With increasing dilution factor, the internal standard responses of the QC samples increased steadily from 1.5 times to 10 times and then stayed relatively constant beyond that. The data matched very well with those shown in Fig. 3. In fact, the average IS responses for QC samples diluted 10 times or beyond were similar to those of plasma standards that were prepared in control plasma. 
a

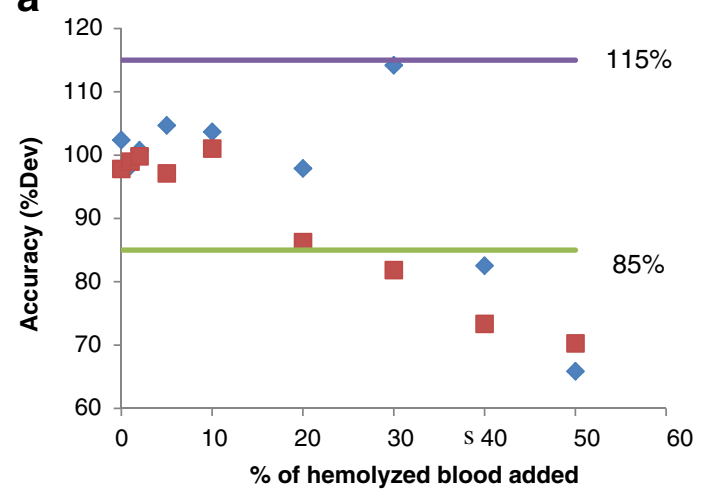

b

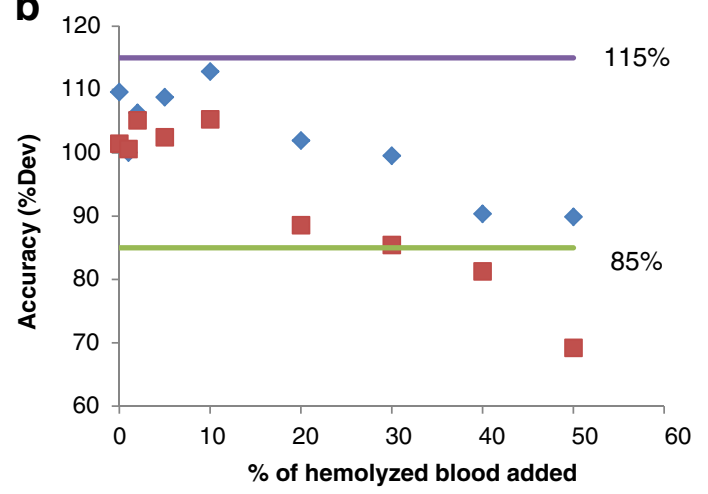

Fig. 4. Average accuracy of triplicate analysis of QC samples for a BMS-906024, b BMS-911557 with different percentages of hemolyzed blood spiked to the plasma. Low conc. is $1.5 \mathrm{ng} / \mathrm{mL}$ and high conc. is $187.5 \mathrm{ng} / \mathrm{mL}$

\section{DISCUSSION}

\section{Impact of Hemolysis on the Reported Bioanalytical Results}

In order to provide a quantitative evaluation of ion suppression/enhancement caused by hemolysis on the analyses of BMS-906024 and BMS-911557, we have chosen the peak areas of their internal standards, BMS-906024-03 and BMS-911557-02 (IS responses) as the surrogate markers. With increasing percentages of hemolyzed blood, the average IS responses from triplicate analysis of the QC samples decreased from $1 \%$ of hemolyzed blood to $20 \%$ of hemolyzed blood, indicating increasing degree of ion suppression on the compounds from the additional endogenous components, with the sharpest drop between $5 \%$ of hemolyzed blood and $20 \%$ of hemolyzed blood. The results were surprising, given that liquid-liquid extraction, which is known to produce cleaner extracted samples for LC-MS/MS analysis than protein precipitation (15), was used in this assay and it failed to sufficiently remove the interfering components when the percentage of hemolyzed blood reached $20 \%$ or beyond. It is also interesting to note that at $2 \%$ of hemolyzed blood, which is the level commonly used for evaluation of matrix factor in hemolyzed sample, the internal standard responses is similar to those of control plasma, indicating that there was minimal matrix effect caused by $2 \%$ of hemolyzed blood. This is supported by the results of an independent evaluation of matrix factor in $2 \%$ of hemolyzed blood in which they yielded a

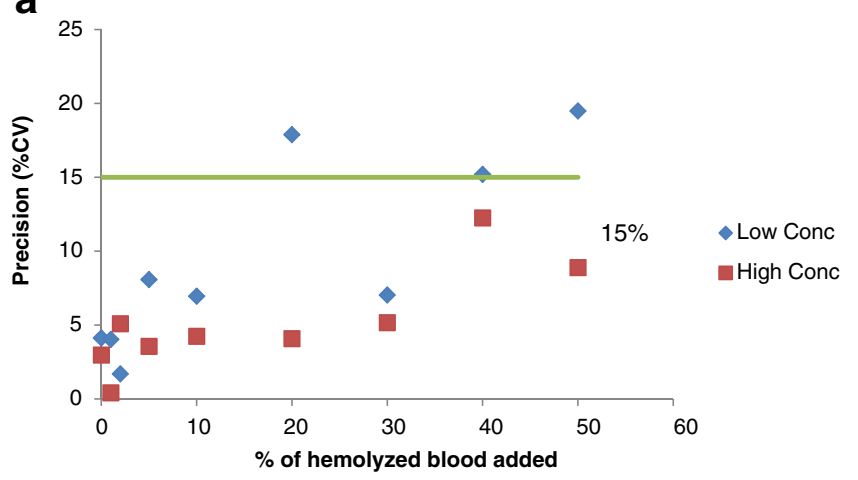

b

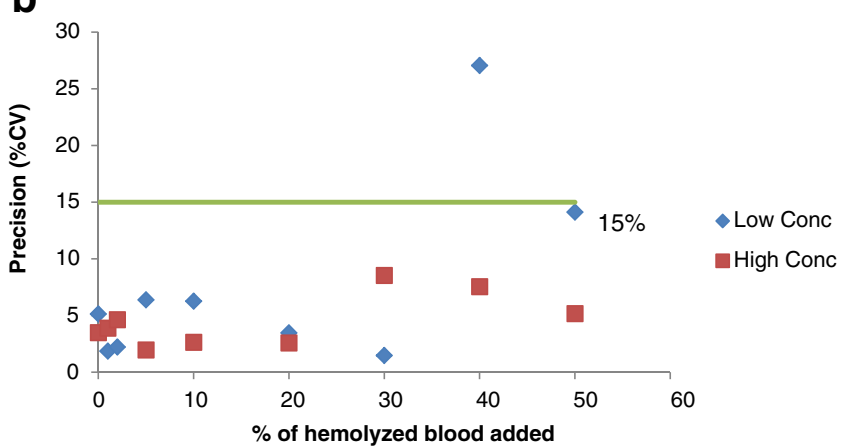

Fig. 5. Precision of triplicate analysis of QC samples for a BMS906024, b BMS-911557 with different percentages of hemolyzed blood spiked to the plasma. Low conc. is $1.5 \mathrm{ng} / \mathrm{mL}$ and high conc. is $187.5 \mathrm{ng} / \mathrm{mL}$

the absolute matrix factor and IS-normalized matrix factor of 0.81 and 0.99 , respectively.

While it may be impossible to totally eliminate matrix effect, SIL-IS such as BMS-906024-03 and BMS-911557-02 in general compensate for the matrix effect on their respective analytes, resulting in good accuracy and precision on the reported concentration of analytes in matrices such as plasma with slight hemolysis (e.g., 1-2\% of hemolyzed blood). However, the same may not be true for severely hemolyzed samples, as demonstrated from the results shown in Figs. 4 and 5. For both BMS906024 and BMS-911557, starting at a concentration of $\sim 20 \%$ of hemolyzed blood, the method showed poorer accuracy and precision. It is worthwhile to note that starting from $20 \%$ of hemolyzed blood, the peak areas of the analytes of the low QC sample $(<600 \mathrm{cps})$ were lower than those at the lower limit of quantitation (LLOQ) ( $2500 \mathrm{cps})$. The very low analyte/ internal standard peak areas which were close to that of the background noise resulted in lower precision and accuracy. This will have significant impact on the analyses of study samples, especially the ones with concentrations close to the LLOQ.

It should be noted that the hemolyzed plasma may not only affect the ionization suppression but also affect the extraction recovery. The work here focuses on the effect of hemolysis. The decrease in IS response for severe hemolyzed plasma could also be due to ionization suppression or recovery loss, or both. Nonetheless, IS response is a good surrogate marker to flag out study samples for further investigation or determine "reportability" of the bioanalytical data generated regardless of the underlying causes. 

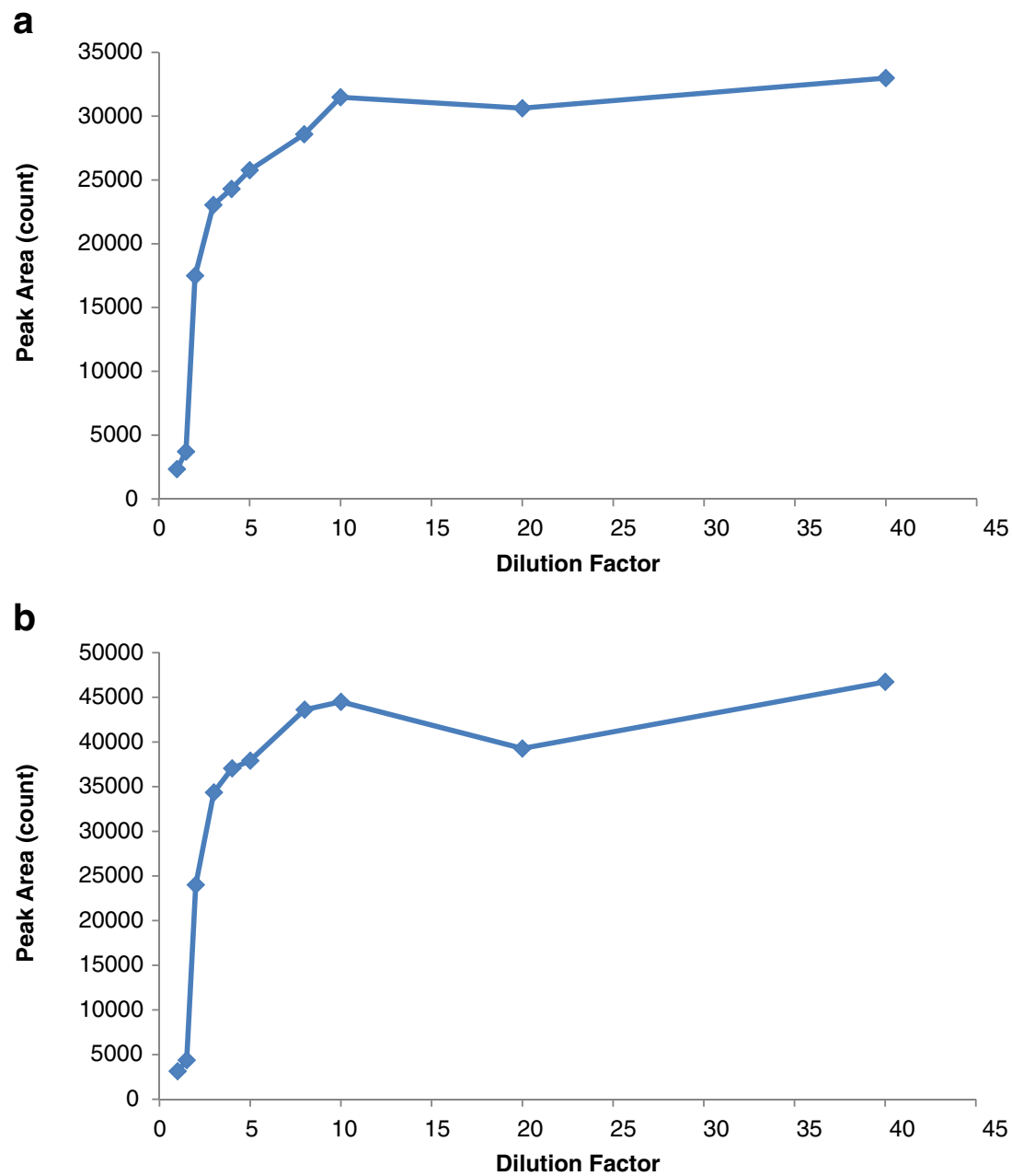

Fig. 6. Average peak area of the internal standards (internal standard responses) of a BMS-906024, b BMS-911557 with different dilution factors from the triplicate analysis of a high concentration QC

In addition, Tan et al. (28) reported that hemolysis during sample collection could have additional impact on the reported drug concentrations depending on the degree of the distribution of analytes between red blood cell and plasma and it is independent of the matrix effect. In these cases, special care should be made on reporting bioanalytical data from this type of compounds.

It would be worthwhile to note that in this work, the hemolyzed plasma was prepared by mixing human plasma with appropriate amount of lysed human whole blood. However, the human whole blood may contain different amount of red blood cells depending on the donor's physiological conditions (e.g., hematocrit). We would suggest that for controlled experiments such as method validation, the hematocrit of the blood should be documented and even standardized in the future.

\section{Effect of Dilution on the Internal Standard Responses of the Bioanalytical Assay}

With the additional matrix effect from endogenous components released from hemolysis, and their detrimental influence on the bioanalytical method in mind, we then investigated a potential resolution to reduce matrix interferences. One obvious solution was to refine the bioanalytical method, e.g., by modifying the extraction method. The major drawback for this is that it requires some degree of re-validation of the bioanalytical method and/or cross-validation between "old" and "new" methods, which is not desirable in the midst of a study.

We turned our attention to dilution of the hemolyzed samples by control plasma. As seen in Fig. 3, the IS responses decreased with increasing percentage of hemolyzed blood. We theorized that we could "in effect" reduce the percentage of hemolyzed blood, and hence matrix effect, by simply diluting hemolyzed samples with control plasma. The results depicted in Fig. 6 clearly showed that the average IS responses increased, and hence the matrix effect decreased, with higher dilution factor. The average IS responses plateaued at a 10-fold dilution, which produced an effective percentage of hemolyzed blood in the diluted samples of $4 \%$. Based on the results from Figs. 4 and 5, the accuracy and precision of the diluted QC samples (at $4 \%$ of hemolyzed blood) should be well within the acceptance criteria. In practice, the severely hemolyzed samples can be analyzed with dilution, if appropriate, to obtain bioanalytical results with acceptable precision and accuracy. 
The Proposed Strategy to Mitigate the Risk of Reporting Bioanalytical Results from Hemolyzed Samples

The susceptibility of the analytes to additional matrix effect caused by hemolysis is certainly dependent on the analytes and bioanalytical method. It is highly desirable to evaluate matrix factor in severely hemolyzed plasma during method development and to develop a method that alleviates the effect of severe hemolysis. However, this may not be feasible or cost-effective given that the likelihood of occurrence of severely hemolyzed samples could be less than $5 \%$ of the total number of samples (22). It is, therefore, preferable to have an objective and quantitative approach to flag these severely hemolyzed samples during sample analysis, especially for the methods that are sensitive to additional matrix effect caused by hemolysis, and to assess the ruggedness of reported bioanalytical results. Theoretically, it is possible to evaluate the extent of hemolysis in each study sample. In practice, there is no steadfast means to determine the percentage of hemolyzed blood in a given study sample. While it is possible to compare the color of the samples with a diagram such as Fig. 2, it is not easy to accurately determine the percentage, e.g., between 10 and $20 \%$. It is also impractical to measure the extent of hemolysis spectrophotometrically for every study sample.

Herein, we propose a strategy to flag the samples that are affected by severe hemolysis by using the IS response as the surrogate marker, as shown in Fig. 7. If the IS response of a severely hemolyzed sample is outside of a pre-defined limit, e.g., less than $50 \%$ or more than $150 \%$ of the average IS response in study samples, plasma standards and QC samples when SIL-IS is used, then the sample is re-analyzed, preferably with multiple dilutions if sufficient amount of sample is available. The IS responses from the re-analysis will be evaluated. If they are comparable to that of the mean IS response of the run (e.g., within $\pm 20 \%$ ), indicating minimal matrix effect after sample dilution, then the measured

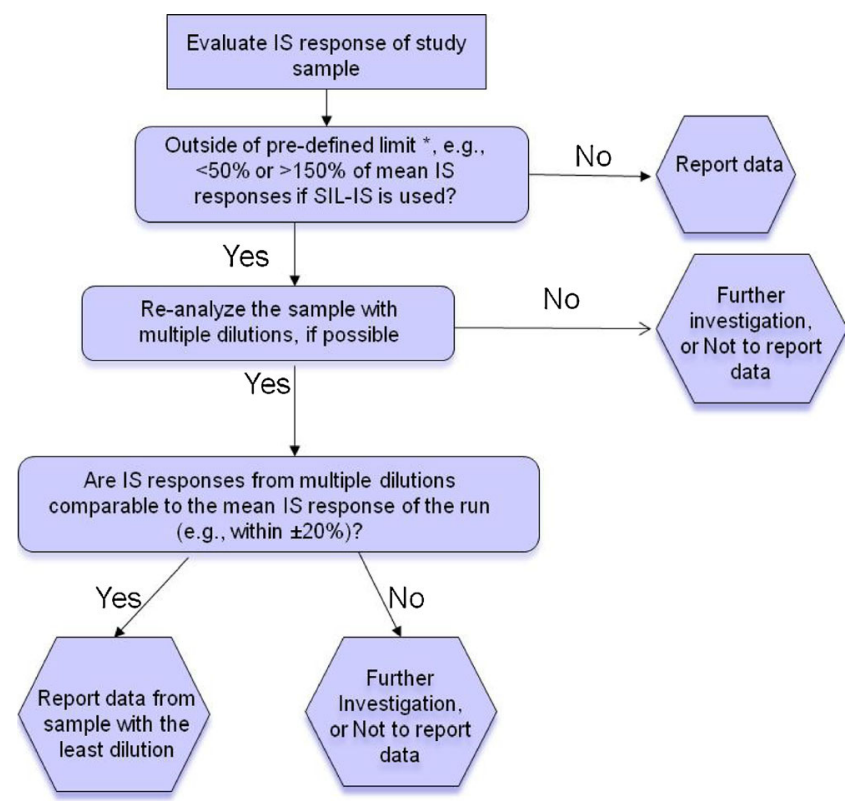

Fig. 7. The proposed strategy to use internal standard responses on reporting bioanalytical data from hemolyzed samples concentration with the least dilution is reported. If there is significant observed variability in IS responses from multiple dilutions, then further investigation, including additional dilutions will be warranted. If it is not possible to re-analyze with dilution (e.g., predicted concentration close to LLOQ), the bioanalysts will further investigate to determine the path forward, such as not reporting the result or changing the bioanalytical method. In practice, study samples can be analyzed and the hemolysis status of the samples is noted in laboratory record, followed by evaluation of IS response upon completion of mass spectrometric analysis. If the IS response of a sample does not meet pre-defined acceptance criteria and the sample is noted as hemolyzed, then the bioanalyst can re-analyze the sample with appropriate dilution. If its concentration is too low for dilution, the bioanalytical data will not be reported.

It is important to note that the effect of hemolysis on the internal standard response and the pre-defined limit are dependent on the particular analyte and the bioanalytical method. For example, if an analog internal standard is used, a pre-defined limit of less than $50 \%$ or more than $150 \%$ of the average IS response may not be appropriate, since an analog internal standard may not track the mass spectrometric behavior of the analyte as well as a stable isotope-labeled internal standard. This pre-defined limit can be specific for hemolyzed samples or a general one for any study samples. The pre-defined limit should be established during method development and validation stage. In the case of our model compounds, we have adopted the acceptance criterion of less than $50 \%$ of the average IS response in all study samples, plasma standards, and QC samples based on the analysis of accuracy and precision of QC samples across the range of hemolyzed blood. The criterion was chosen based on the results depicted in Figs. 4 and 5, in which the accuracy and precision still met the regulatory acceptance criteria at $\sim 10 \%$ of hemolyzed blood. This corresponded to less than $50 \%$ decrease of average IS response as shown in Fig. 3. The authors have used this strategy to flag study samples in multiple clinical studies, in which the occurrence of hemolyzed samples is about $4.6 \%$ of the study samples and successfully implemented the dilution approach with less than $0.2 \%$ of non-reportable results.

Nowadays, a lot of bioanalytical laboratories routinely flag the samples with internal standards outside of a predefined limit for further evaluation. The proposed strategy can be readily incorporated and implemented in routine sample analysis for hemolyzed samples and should not be any additional burden. It also provides a quantitative criterion to evaluate the effect of hemolysis on the bioanalytical results, especially in cases in which bioanalytical results cannot be reported, e.g., samples with extremely low IS response and sample dilution is not possible due to low concentration.

For best practices, it is highly desirable for the bioanalyst to apply the strategy to carefully evaluate the effect of hemolysis on accuracy, precision, and internal standard peak area of the method during method development. It is suggested to conduct a series of experiments by analyzing a series of QC samples prepared in different percentage of hemolyzed blood, using plots similar to Figs. 3, 4, and 5 to establish the pre-defined limit during method development and validation. It is prudent to evaluate a wide range of 
percentages of hemolyzed plasma, as much as 20 to $50 \%$ even though it is rare to have study samples with more than $20 \%$ of hemolyzed blood, instead of merely focusing around 2 to $5 \%$ because the study samples can represent more significant levels of hemolysis. If the IS response changes (e.g., lower than 50\%) do not impact on accuracy (in other words, the internal standard can normalize the hemolyzed effect), then the method can be used to analyze study samples. In cases where hemolysis impacts the accuracy and precision of the method, additional method development work (e.g., changing sample extraction procedures) will be warranted to eliminate the undesirable matrix effect. If it is not feasible or cost-effective to eliminate the undesirable matrix effect, it is suggested to determine at what percentage of hemolyzed blood that the IS response changes sharply, coupled with lower accuracy and precision (breaking point). This serves as a guide to the limitation of the method. Study samples are compared with the "breaking point," and samples that appear to be more hemolyzed than that of the breaking point can then be analyzed with appropriate dilution. This will minimize the need of additional re-analysis of severely hemolyzed samples.

\section{CONCLUDING REMARKS}

In this work, we demonstrated the effect of severe hemolysis on the accuracy and precision of a LC-MS/MS-based bioanalytical method, which is a direct result of additional matrix effect on the ionization of the analytes caused by the endogenous material released from the hemolysis. The peak area of the internal standard (IS response) is proposed to be used as a universal and quantitative mean to assess the effect of hemolysis on the reported bioanalytical results from individual study samples. Study samples with an IS response outside of the acceptance criteria will be re-analyzed with dilution, if possible, to obtain reportable results. If re-analysis of the flagged study samples fails to meet acceptance criteria, the bioanalysis will further investigate to determine a path forward, including refining the assay or not reporting the bioanalytical data for the flagged study samples.

The strategy can also be adopted for the analysis of hyperlipemic samples or any other type of variable matrix effect. Lipids, such as phospholipids, are present in plasma, and it has been demonstrated that they cause significant matrix effect $(29,30)$. Evaluation of matrix factor and selectivity in hyperlipemic samples has been routinely conducted in bioanalytical laboratories. Based on the authors' experience, a decrease in IS response has been observed in hyperlipemic samples as well and diluting the samples in questions resulted in improvement in internal standard response. There are a number of clinical parameters resulting in hyperlipemia such as cholesterol and triglyceride (31); further work is needed to determine which ones would have the most significant impact on the analyte(s) of interest.

\section{ACKNOWLEDGMENTS}

The authors would like to thank Drs. Mark E. Arnold, Bruce S. Fischer, Michael Tagen, and Mrs. Ryan KirchoffRoss for their helpful discussions and Mr. William Cotton for technical support.
Conflict of Interest The authors are current employees of BristolMyers Squibb Company (BMS). All financial support for the studies reported herein was provided by BMS. The authors have no further relevant affiliations or financial involvement with any other organization or entity with a financial interest in or financial conflict with the subject matter or materials discussed in the manuscript. This includes consultancies, honoraria, stock ownership or options, expert testimony, grants or patents received or pending, or royalties. No writing assistance was utilized in the production of this manuscript.

\section{REFERENCES}

1. Maurer HH. Current role of liquid chromatography-mass spectrometry in clinical and forensic toxicology. Anal Bioanal Chem. 2007;388:1315-25.

2. Xu RN, Fan L, Rieser MJ, El-Shourbagy TA. Recent advances in high-throughput quantitative bioanalysis by LC-MS/MS. J Pharm Biomed Anal. 2007;44:342-55.

3. Korfmacher WA. Foundation review: principles and applications of LC-MS in new drug discovery. Drug Discov Today. 2005;10:1357-67.

4. Li W, Tse FLS. Dried blood spot sampling in combination with LC-MS/MS for quantitative analysis of small molecules. Biomed Chromatogr. 2010;24:49-65.

5. Srinivas NR, Mullangi R. An overview of various validated HPLC and LC-MS/MS methods for quantitation of drugs in bile: challenges and considerations. Biomed Chromatogr. 2011;25:65-81.

6. van den Broek I, Niessen WM, van Dongen WD. Bioanalytical LC-MS/MS of protein-based biopharmaceuticals. J Chromatogr B Analyt Technol Biomed Life Sci. 2013;929:161-79.

7. Liang Z. Perspectives on addressing ionization matrix effects in LC-MS bioanalysis. Bioanalysis. 2012;4:1227-34.

8. Liu G, Ji QC, Arnold ME. Identifying, evaluating, and controlling bioanalytical risks resulting from nonuniform matrix ion suppression/enhancement and nonlinear liquid chromatographymass spectrometry assay response. Anal Chem. 2010;82:9671-7.

9. Larger PJ, Breda M, Fraier D, Hughes H, James CA. Ionsuppression effects in liquid chromatography-tandem mass spectrometry due to a formulation agent, a case study in drug discovery bioanalysis. J Pharm Biomed Anal. 2005;39:206-16.

10. Dams R, Huestis MA, Lambert WE, Murphy CM. Matrix effect in bio-analysis of illicit drugs with LC-MS/MS: influence of ionization type, sample preparation, and biofluid. J Am Soc Mass Spectrom. 2003;14:1290-4.

11. Fung EN, Zheng N, Arnold ME, Zeng J. Effective screening approach to select esterase inhibitors used for stabilizing estercontaining prodrugs analyzed by LC-MS/MS. Bioanalysis. 2010;2:733-43.

12. King R, Bonfiglio R, Fernandez-Metzler C, Miller-Stein C, Olah $\mathrm{T}$. Mechanistic investigation of ionization suppression in electrospray ionization. J Am Soc Mass Spectrom. 2000;11:942-50.

13. Matuszewski BK, Constanzer ML, Chavez-Eng CM. Strategies for the assessment of matrix effect in quantitative bioanalytical methods based on HPLC-MS/MS. Anal Chem. 2003;75:3019-30.

14. Matuszewski BK. Standard line slopes as a measure of a relative matrix effect in quantitative HPLC-MS bioanalysis. J Chromatogr B Analyt Technol Biomed Life Sci. 2006;830:293-300.

15. Van Eeckhaut A, Lanckmans K, Sarre S, Smolders I, Michotte Y. Validation of bioanalytical LC-MS/MS assays: evaluation of matrix effects. J Chromatogr B. 2009;877:2198-207.

16. Viswanathan CT, Bansal S, Booth B, DeStefano AJ, Rose MJ, Sailstad J, et al. Workshop/conference report-quantitative bioanalytical methods validation and implementation: best practices for chromatographic and ligand-binding assays. AAPS J. 2007;9:E30-42.

17. ANVISA. Resolution RDC No.27,17 May 2012. Brazil: Brazilian Health Surveillance Agency; 2012.

18. European Medicines Agency. Guideline on bioanalytical method validation, London, UK, committee for medicinal products for 
human use (2011) FDA, guidance for industry: bioanalytical method validation; 2011.

19. Hughes NC, Bajaj N, Fan J, Wong EY. Assessing the matrix effects of hemolyzed samples in bioanalysis. Bioanalysis. 2009;1:1057-66.

20. Bérubé ER, Taillon MP, Furtado M, Garofolo F. Impact of sample hemolysis on drug stability in regulated bioanalysis. Bioanalysis. 2011;3:2097-105.

21. Garofolo F, Rocci Jr ML, Dumont I, Martinez S, Lowes S, Woolf $\mathrm{E}$, et al. 2011 White paper on recent issues in bioanalysis and regulatory findings from audits and inspections. Bioanalysis. 2011;3:2081-96.

22. Tang D, Thomas E. Strategies for dealing with hemolyzed samples in regulated LC-MS/MS bioanalysis. Bioanalysis. 2012;4:2715-24.

23. Ingelse B, Barroso B, Gray N, Jakob-Rodamer V, Kingsley C, Sykora $\mathrm{C}$, et al. European bioanalysis forum: recommendation on dealing with hemolyzed and hyperlipidemic matrices. Bioanalysis. 2014;6:3113-20.

24. Remane D, Wissenbach DK, Meyer MR, Maurer HH. Systematic investigation of ion suppression and enhancement effects of fourteen stable-isotope-labeled internal standards by their native analogues using atmosphericpressure chemical ionization and electrospray ionization and the relevance for multi-analyte liquid chromatographic/ mass spectrometric procedures. Rapid Commun Mass Spectrom. 2010;24:859-67.

25. Berg T, Strand DH. 13C labelled internal standards-a solution to minimize ion suppression effects in liquid chromatography- tandem mass spectrometry analyses of drugs in biological samples? J Chromatogr A. 2011;1218:9366-74.

26. Gavai AV, Quesnelle C, Norris D, Han WC, Gill P, Shan W, et al. Discovery of Clinical Candidate BMS-906024: A Potent PanNotch Inhibitor for the Treatment of Leukemia and Solid Tumors. ACS Med Chem Lett. 2015. doi:10.1021/ acsmedchemlett.5b00001.

27. Food and Drug Administration. Guidance for industry: bioanalytical method validation. Rockville: US department of Health and Human Services, FDA. Center for Drug Evaluation and Research; 2001.

28. Tan A, Gagné S, Lévesque IA, Lachance S, Boudreau N, Lévesque A. Impact of hemolysis during sample collection: how different is drug concentration in hemolyzed plasma from that of normal plasma? J Chromatogr B Analyt Technol Biomed Life Sci. 2012;901:79-84.

29. Xia YQ, Jemal M. Phospholipids in liquid chromatography/mass spectrometry bioanalysis: comparison of three tandem mass spectrometric techniques for monitoring plasma phospholipids, the effect of mobile phase composition on phospholipids elution and the association of phospholipids with matrix effects. Rapid Commun Mass Spectrom. 2009;23:2125-38.

30. Clark GT, Russell PJ, Westwood S. Modification without impact: a case study in clinical assay failure due to lipemia. Bioanalysis. 2012;4:1421-8.

31. Mayrand-Provencher L, Furtado M, Mess JN, Dumont I, Garofolo F. Choosing the appropriate matrix to perform a scientifically meaningful lipemic plasma test in bioanalytical method validation. Bioanalysis. 2014;6:1639-46. 